International Journal of Pure and Applied Mathematics

Volume 93 No. 3 2014, 399-408

ISSN: 1311-8080 (printed version); ISSN: 1314-3395 (on-line version)

url: http://www.ijpam.eu

doi: http://dx.doi.org/10.12732/ijpam.v93i3.10

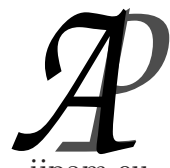

ijpam.eu

\title{
THE MATHEMATICS OF BENHAM'S TOP
}

\author{
Yutaka Nishiyama \\ Department of Business Information \\ Faculty of Information Management \\ Osaka University of Economics \\ 2, Osumi Higashiyodogawa Osaka, 533-8533, JAPAN
}

\begin{abstract}
It is still a mystery why colors emerge from the black and white pattern of Benham's top. After a brief overview of previous research on subjective colors, this article explains the nature of color and how we humans perceive a color. This article also introduces a hypothesis about subjective colors based on neuronal stimulation, the characteristics of sensation, and in particular neural transmission delay.
\end{abstract}

AMS Subject Classification: 92C20, 00A08, 94A02

Key Words: Benham's top, subjective color, color, neuron, stimulation and sensation, threshold characteristics and delay

\section{Appearance of Seven Colors from Black and White}

Some of you might have heard about Benham's top, a mysterious spinning disk created by Mr. Benham (Fig. 1). Half the top is painted in black and the other half is white with several pairs of 3 arcs. Do you know what happens when you spin this disk? Every year I introduce Benham's top to students in my information mathematics class.

Although you might expect to see black or gray lines because of the black painting on the white background, in reality colors such as red, blue, and green

Received: January 18, 2014

(c) 2014 Academic Publications, Ltd. url: www.acadpubl.eu 
will appear. When I say this, students do not believe it, thinking that it is a sort of magic trick. It is also questionable how well I can explain the phenomenon to readers without actual showing you it, but I will do my best to explain the concept behind this spinning disk.

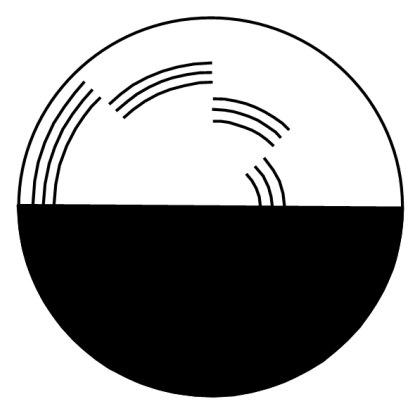

Figure 1: Benham's top

When a disk like the one shown above is spun around forcefully, nothing shows up at first, but as the speed decreases and the pattern on the top starts to flicker, colors suddenly emerge. When rotating counterclockwise, red, orange, green, and blue colors appear, with red being the outermost color (Fig. 2a). Although these colors are not vivid, they are undoubtedly not black or gray. When rotating clockwise, interestingly the disk produces blue, green, orange, and red colors, with blue being the outermost color (Fig. 2b); thus, the order of colors is opposite. I will explain the reason below.

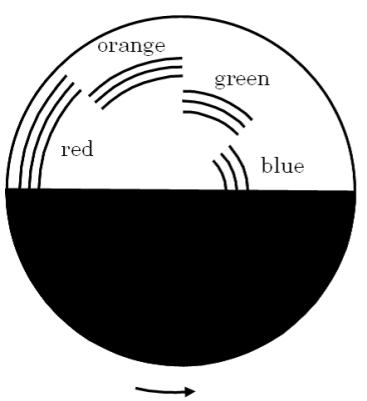

(a) Counterclockwise rotation

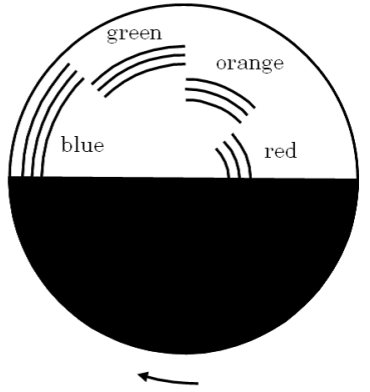

(b) Clockwise rotation

Figure 2: The order of colors differs depending on the direction of rotation. 


\section{Study of Subjective Colors}

The human eye can see achromatic colors when white light is shown intermittently or a disk with an alternating pattern of black and white is spinning. Because this phenomenon is unpredictable due to the spectral characteristics of the stimuli, these colors are called subjective colors or Fechner's colors, the latter of which bears the name of the principal discoverer of the phenomenon.

Studies of subjective colors conducted before 1948 are described in detail in an article by J. Cohen and D. A. Gordon [1]. Subjective colors were first discovered by a French monk, Prévost, in 1826. While observing a beam of white light in a dim room, he intermittently blocked the light beam by moving a piece of white rectangular cardboard perpendicular to the light. Although seeing only white at first, he started to observe purple in the center, followed by deep indigo, red, and yellow. Prévost described the phenomenon as being similar to decomposing white light into basic colors using a prism, although he never meant that the light was actually decomposed as Newton had previously demonstrated. Prévost thought that there must be special molecules in the retina that evoke neural sensation associated with colors and that these molecules would react to the light at different rates. At that time, the concept of three types of color receptors had not yet been established.

Subjective color was rediscovered by Fechner in 1839. Fechner created disks with an opposite black and white image as shown in Figure 3a and b. When the disks are spun, they show a different degree of gray because of the difference in the black to white ratios. As the speed decreases and the light starts to flicker, because of reaching the critical fusion frequency, many colors appear. The colors are not vivid, but they are clearly colors. Fechner explained that the speed and direction of rotation are the major determinants of subjective colors.

Subjective colors were also observed in 1848 by Dove and in 1859 by John Smith using a disk shown in Figure 3c and d, respectively. In 1860, Helmholtz attempted to apply a scientific principle to explain subjective colors and quoted Maxwell's disk in his study. Helmholtz adopted the theory of Fechner and hypothesized that the phenomenon was caused by differences in the rise and fall time of the retinal photoreceptors specific to red, green, and blue, with blue light receptors responding more slowly than those for red. Figure $3 \mathrm{e}$ and $\mathrm{f}$ show a disk created by Helmholtz and Brücke, respectively.

In England in 1894 to 1895, people were fascinated by a disk called gan artificial spectrum toph. This disk was also known as gBenham's toph and was discussed in the British scientific journal Nature. It was extremely popular and 


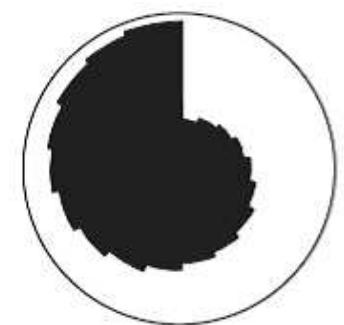

(a) Fechner

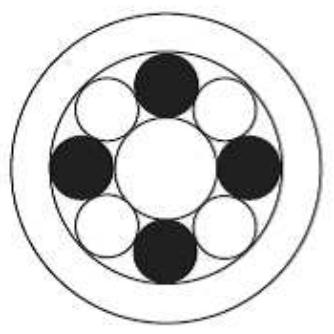

(c) Dove

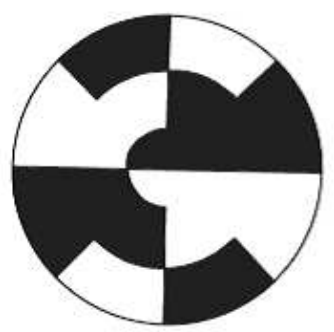

(e) Helmholtz

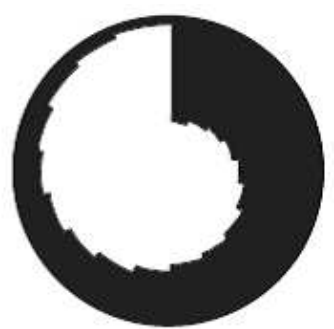

(b) Fechner

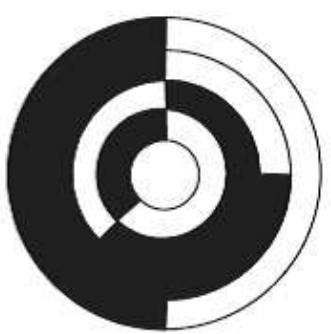

(d) John Smith

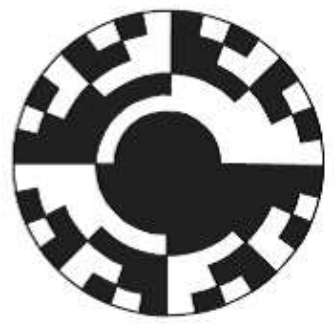

(f) Brücke

Figure 3: Spinning disks with a pattern that generates subjective colors (see [1]) 
sold widely as a toy. What made this disk interesting as a toy was the fact that colors appear in the same order as the decomposed colors from a prism.

Since then, numerous researchers have created a disk that produces subjective colors and have hypothesized the cause of these colors. However, it has been difficult to confirm their theories and many uncertainties remain because the phenomenon is deeply intertwined with the functional properties of the human eye, optical nerve, and central nervous system.

\section{Light: From the Eyes to the Cerebrum}

The question of why we perceive color has been one of the most researched topics in the last 200 years since the days of Goethe and Newton. In physics, colors are visible light to the human eye, and their electromagnetic waves in the air range from approximately 380 to $780 \mathrm{~nm}$.

Things are not visible when the light is off because the human eye needs light to perceive a color. If asked what color the light is, we must answer that the light contains not only white, but all colors. In addition, leaves appear green because only the green wavelength is reflected and other wavelengths, such as blue and red, are absorbed.

However, we sometimes see colors without light. When the eyes are closed and the eyelids are pressed with the fingers, a blue spherical object appears in a reddish purple background. Some people dream in color, but this must be caused by certain activities in the cerebrum because no stimuli are present to activate the retina during sleep.

The organ that senses colors in our body is the eye, and it can be considered a sophisticated automatic camera. The lens is crystal and the surrounding muscles involuntarily adjust the curvature of the lens, focusing the light entering the eyes and creating a clear image on the retina which takes the role of a film and a dry plate. The retina converts the light energy into electrical pulses and transmits the information to the brain through the optic nerve. In the outermost layer of the retina, rod photoreceptor cells are sensitive to dim light and cone cells are responsive for color vision. While rods are present throughout the retina, cones are densely packed only in the central portion of the retina. Their distribution there makes colors vivid in bright conditions, making the pupil contract; only achromatic contrast is detected as it gets dark and the pupil dilates.

In 1964, Marks et al. used sophisticated equipment to successfully microanalyze a single cone cell. After discovering that cone photoreceptor cells in 
goldfish retina absorb red $(625 \mathrm{~nm})$, green $(530 \mathrm{~nm})$, and blue $(455 \mathrm{~nm})$ light, he also found the absorption of three distinct wavelengths, 570, 535, and 455 $\mathrm{nm}$ in monkeys and humans.

Visual information from the two-dimensional array of retinal photoreceptor cells is transmitted simultaneously to the higher order neurons in a parallel fashion. However, the numbers of optic nerves connecting the retina to the cerebrum are far fewer than those of retinal photoreceptor cells, and so there is no 1-to-1 correspondence between them. Moreover, the speed of the visual phototransduction depends on the route. Because the neural network from the retina to the optic nerve is a three-dimensional temporal and spatial circuit, this not only complicates the perception of color, but also contributes to the appearance of subjective colors.

\section{Stimulation and Characteristics of Sensations}

Neurons are the basic elements of neural information processing system in our body, and the basic structure of a neuron is shown in Figure 4. The main body of a neuron is the cell body, or soma, which gives rise to multiple dendrites and an axon. The axon travels a long distance to convey the neuronal excitation to its target neuron(s). In general, there are several branches at the axonal terminal, and each branch has an interface called a synapse to interact with another neuron.

Ion conduction is the primary signal transduction system inside a neuron. A high concentration of potassium $(\mathrm{K})$ ions is present inside an axon, while the concentration of sodium $(\mathrm{Na})$ ions is high outside the cell membrane. At rest (without excitation), the resting potential inside the plasma membrane is negative and a couple of tens of $\mathrm{mV}$ lower than that outside. When excited, the plasma membrane becomes selectively permeable to $\mathrm{K}^{+}$and $\mathrm{Na}^{+}$, allowing the outward and inward flow of $\mathrm{K}^{+}$and $\mathrm{Na}^{+}$ions, respectively. This triggers the membrane potential inside the neuron to increase and become positive. This positive potential is called the action potential. The excitation results in a domino-like propagation of action potentials through the axonal plasma membrane. Action potentials propagate through the patches of excitable membrane, which is permeable to $\mathrm{K}^{+}$and $\mathrm{Na}^{+}$ions, and are not generated by the actual movement of these ions inside the axon.

Figure 5 shows the changes in the sensory perception of light when light with intensity $I$ is turned on and off at time 0 and $T=t_{5}$, respectively.

Stimuli and sensations do not necessarily correspond in time and magnitude. 


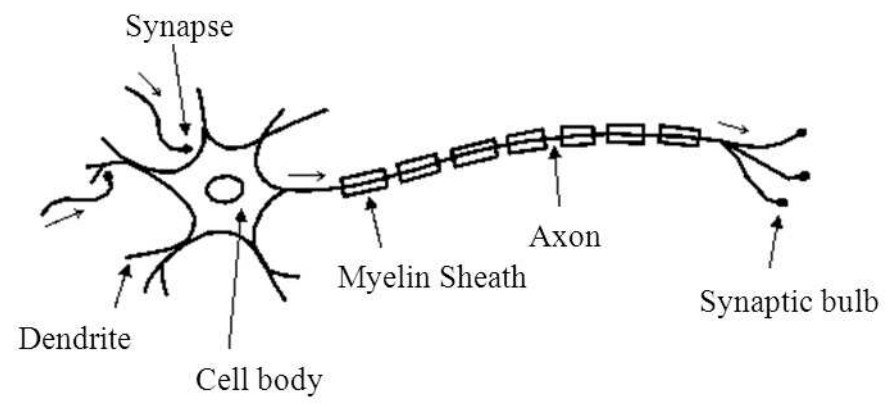

Figure 4: The basic structure of a neuron

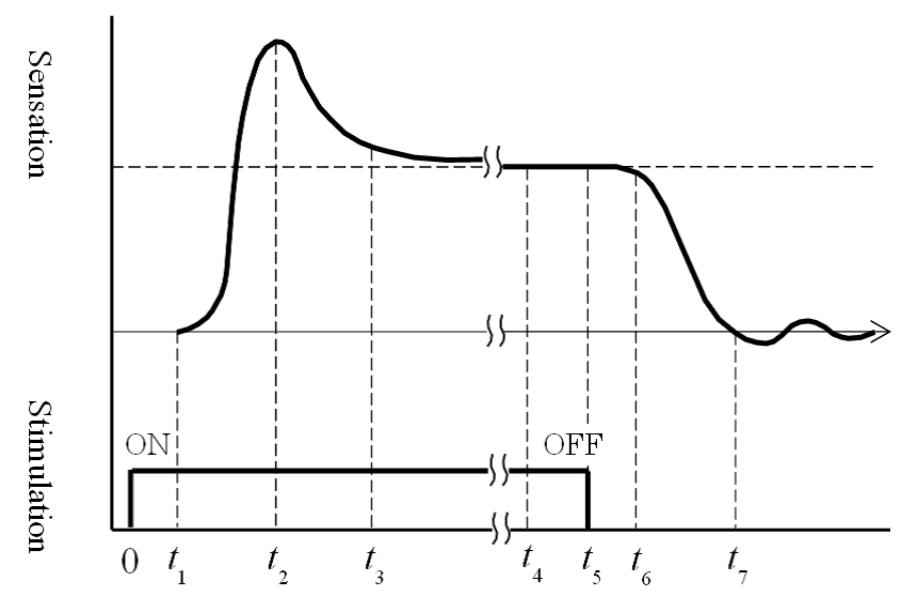

Figure 5: Elapsed time between light stimulation and sensation (see [2]) 
A sensation follows stimulation with a slight delay and eventually enters a state of adaptation. Sensation remains even after sensory stimulation has stopped.

Such psychological phenomena are thought to be associated with the ion conduction system among neurons. The presence of stimulus threshold, which prevents a subthreshold stimulus from generating an action potential, and neural transmission delay are unique to neurons, and these neuron-specific characteristics may explain the illusion of subjective colors.

\section{Hypothesis}

I once tried to capture the colors emerging from Benham's top. But of course, the colors did not show up on the photo, which instead showed just slurred black and white lines. It was not due to the shutter speed: it was fundamentally wrong to regard subjective colors as those in the optical system, as subjective colors are a creation of the human visual system. This led me to develop a hypothesis.

I changed the circular pattern of Benham's top (Fig. 1) into a linear one as shown in Figure 6a. You can see that half of the top is black and the other half is white with four pairs of three lines. In its modified version (Fig. 6b), the outer (top) and the inner (bottom) pairs of lines are no longer in contact with the black area or line.

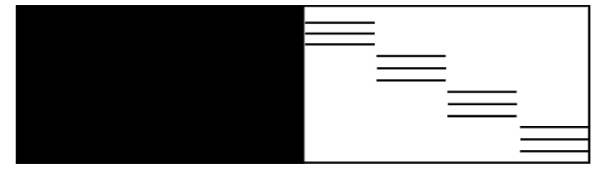

(a)

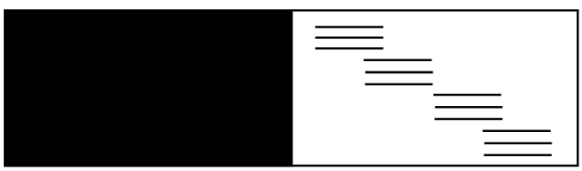

(b)

Figure 6: Conversion from a circular to a linear pattern

The black area on the left is not involved in the generation of color and can be used to rest your eyes. Let's focus on the other half on the right. Each pair of lines divides the white area into two segments. Because the area in 
white reflects light and the one in black absorbs it, the illusion of subjective colors caused by Benham's top is a phenomenon resulting from a series of two distinct light sensations. With the white segments as a stimulus, the four pairs of lines were converted into the unique pattern shown in Figure 7. Because there are two stimuli for each pair of lines, let's call them the first and the second stimulus. The intensity of these stimuli depends on the pair.

I generated my hypothesis based on the neuronal characteristics described above. Even if the first stimulus activates the retinal photoreceptors, the signal transmission to the optic nerve might be delayed because of the three lines that follow the first stimulus. I assume that the extent of the transmission delay correlates negatively with the intensity of the first light stimulus. The greater the first stimulus (the longer the initial white segment), the shorter the delay. The shorter the stimulus, the longer the delay. This must be similar to the concept of mechanics; it is easier to move a smaller object.

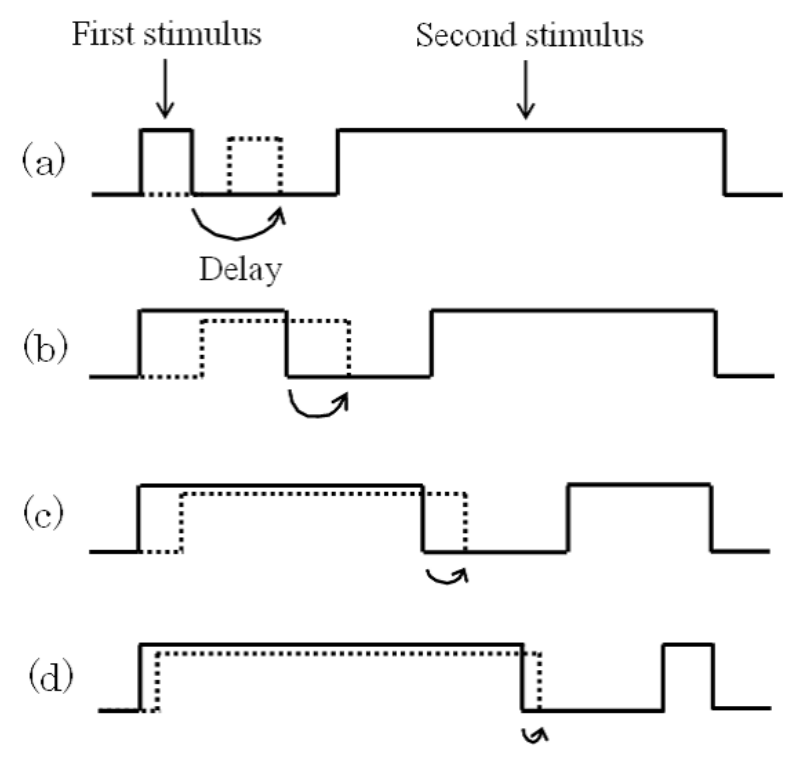

Figure 7: Interference between first and second stimuli

What happens when the second stimulus overlaps the first stimulus shown in a dotted line? Figure 7a-d correspond to red, orange, green, and blue in the order of increasing wavelength. The hypothesis is valid when we assume that only an emphasized wavelength emerges as a color because of the interference between the first and second stimuli. The Newton rings are an objective in- 
terference pattern formed by reflecting light rays; however, the colors emerging from Benham's top are subjective and dynamic. Even today, the cause for subjective colors is not entirely clear. The discovery of an equivalent circuit involved in color vision must be Nobel Prize material [3].

You need to actually see Benham's top to experience subjective colors, and recent advancements in computer technology allow you to do this online [4]. The website introduces you to 107 different optical illusions including Benham's top.

\section{References}

[1] J. Cohen and D. A. Gordon, The Prévost-Fechner-Benham Subjective Colors, Psychological Bulletin, 46(2), (1949), 97-136, doi: 10.1037/h0060841.

[2] K. Hiwatashi, Biological Engineering, Coronasha, Tokyo, (1971).

[3] Y. Nishiyama, The Scent of Science, Nippon Hyoronsha, Tokyo, (1991).

[4] M. Bach, 107 Optical Illusions \& Visual Phenomena by Michel Bach, http://www.michaelbach.de/ot/ 